\title{
Communication \\ Effect of Reciprocating Gait Orthosis with Hip Actuation on Upper Extremity Loading during Ambulation in Patient with Spinal Cord Injury: A Single Case Study
}

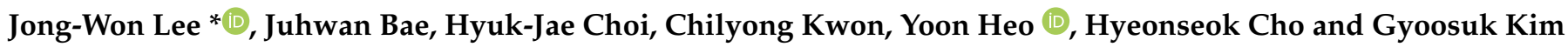

\section{check for} updates

Citation: Lee, J.-W.; Bae, J.; Choi, H.-J.; Kwon, C.; Heo, Y.; Cho, H.; Kim, G. Effect of Reciprocating Gait Orthosis with Hip Actuation on Upper Extremity Loading during Ambulation in Patient with Spinal Cord Injury: A Single Case Study. Machines 2022, 10, 108. https:// doi.org/10.3390/machines10020108 Academic Editors: Chin-Sheng Chen and Tanaka Eiichiro

Received: 9 December 2021

Accepted: 24 January 2022

Published: 29 January 2022

Publisher's Note: MDPI stays neutral with regard to jurisdictional claims in published maps and institutional affiliations.

Copyright: (C) 2022 by the authors. Licensee MDPI, Basel, Switzerland. This article is an open access article distributed under the terms and conditions of the Creative Commons Attribution (CC BY) license (https:// creativecommons.org/licenses/by/ $4.0 /)$.
Rehabilitation Engineering Research Institute, Korea Workers' Compensation \& Welfare Service, Incheon 21417, Korea; juhwan0307@kcomwel.or.kr (J.B.); choi4215@kcomwel.or.kr (H.-J.C.); cykwon@kcomwel.or.kr (C.K.); yoon4888@gmail.com (Y.H.); hscho@kcomwel.or.kr (H.C.); gskim7379@kcomwel.or.kr (G.K.)

* Correspondence: jongwonia@gmail.com
Abstract: Reciprocating gait orthosis (RGO) is a traditional passive orthosis that provides postural stability and allows for independent upright ambulation with the assistance of walking aids, such as crutches, canes, and walkers. Previous follow-up studies of patients with RGOs have indicated a high frequency of nonusage. One of the main reasons for avoiding the use of RGOs is the excessive upper extremity loading induced by walking aids. The purpose of this study was to investigate the effect of hip actuation on the upper extremity loading induced by crutches when ambulating with an RGO. One female individual with a chronic complete spinal cord injury classified as ASIA A participated in this study. We compared the upper extremity loading during ambulation when individualized hip assistive forces were applied on the RGO (POWERED condition) and when wearing the RGO without actuation (RGO condition). Upper extremity loading was assessed by measuring the forces acting on the crutches. Compared with the RGO condition, the average upper extremity loading per unit distance and per unit time were lower for the POWERED condition by $15.21 \%$ (RGO: $0.307 \pm 0.056$ and POWERED: $0.260 \pm 0.034 \% \mathrm{bw} \cdot \mathrm{m}^{-1}$ ) and by $21.19 \%$ (RGO: $0.120 \pm 0.020$ and POWERED: $\left.0.094 \pm 0.011 \% \mathrm{bw} \cdot \mathrm{s}^{-1}\right)$, respectively. We believe that a substantial reduction in upper extremity loading during ambulation provided by hip actuation holds promise to promote long-term RGO use and enable patients with paraplegia to perform frequent and intensive rehabilitation training. As this is a single case study, subsequent studies should aim to verify this effect through a higher number of patients and to different injury levels.

Keywords: reciprocating gait orthosis; hip actuation; spinal cord injury

\section{Introduction}

A spinal cord injury (SCI) is damage to the spinal cord that causes the loss of sensory and motor functions served by the spinal cord below the level of the injury. The essential goals of rehabilitation in patients with SCI are to recover locomotor function, decrease secondary medical complications, and improve health-related quality of life. Regular weight-bearing activities, such as standing and walking, have been reported to reduce the risk of complications due to a lack of time spent in an upright position after SCI, including respiratory disorders, gastrointestinal and cardiovascular disorders, skin and musculoskeletal problems, and psychological disorders [1,2].

To assist patients with SCI in performing weight-bearing activities, various types of passive orthoses have been employed according to the level of SCI and the degree of motor impairment [3]. Among them is reciprocating gait orthosis (RGO), which provides postural stability and enables independent upright ambulation with the assistance of walking aids, such as crutches, canes, and walkers [4-7]. 
An RGO comprises bilateral leg braces coupled to a trunk module with a revolute joint for hip movement in the sagittal plane. Both hip joints are coupled by a reciprocating mechanism using cable or linkage system. This mechanism plays an important role in providing reciprocal ambulation while preventing bilateral hip flexion, which may lead to collapse [8-11]. It has been reported that RGO provides a considerably natural gait with less metabolic energy consumption compared to long leg braces, which do allow for free hip movements [12].

However, follow-up studies on patients with RGOs have indicated a high frequency of nonusage $[7,13,14]$. Among the main reasons for avoiding the use of RGOs listed in previous studies, we focus on the high load applied on the upper extremities during ambulation. When standing and walking with orthoses, paraplegic patients tend to rely more on walking aids than their feet because of their sensorimotor impairment $[15,16]$. Walking aids are used not only to support the body weight to prevent the subject from falling, but also to produce a propulsive force to shift the center of mass (CoM) in the medial-lateral and anterior-posterior directions [17].

Multiple research groups have investigated upper extremity loading in paraplegic patients during ambulation using walking aids [17-20]. A study by Melis et al. showed that the average maximal axial forces exerted on the walking aids ranged from $13 \%$ to $74 \%$ of the body weight during assisted-gait of patients with SCI [17]. These forces depended on the type of walking aids used. Ferrarin et al. reported that the maximum load on the crutches is approximately $30 \%$ of the body weight during paraplegic locomotion with hip guidance orthosis [19]. The findings of Solomonow et al. indicate that RGO users endure high shoulder loads from both crutches and walkers for up to one-third of their body weight [18]. This undesirable load may contribute to upper limb pathologies, such as destructive shoulder arthropathy, degenerative arthritis of the shoulder and wrist, or carpal tunnel syndrome, and in turn hinder the widespread use of RGOs [21]. Because rehabilitative outcomes are largely dependent on training dosage, intensity, and number of repetitions, it is vital to reduce upper extremity loading for long-term RGO use [22,23].

Recently, with advances in robotic technology, powered exoskeletons have been developed to facilitate ambulation in patients with SCI [24]. In contrast to traditional passive orthoses, they can provide well-coordinated joint movements using actuators, sensors, and an embedded control system. Previous published studies have reported that powered exoskeletons improve functional mobility during ambulation in patients with paraplegia [25,26]. In comparative studies, Farris et al. found that walking with a powered exoskeleton provides increase in walking speed and a concomitant decrease in required exertion relative to walking with the knee-ankle-foot orthoses (KAFOs) [27]. Kwon et al. also found that powered exoskeletons enable patients with paraplegia to walk with lower energy consumption compared to KAFOs [28]. In a comparison between RGO and powered exoskeleton, energy expenditure during ambulation was found to be higher in patients with SCI using an RGO than in those using a powered exoskeleton [29].

However, most of powered exoskeletons still require walking aids to assist their balance and for propulsion during ambulation. To the best of our knowledge, no existing studies have investigated the effect of powered exoskeletons on the upper extremity loading induced by walking aids. Thus, the purpose of the present study was to investigate how powering human joints affected the upper extremity loading caused by walking aids. Previous biomechanical analyses indicated that the hip extensor muscles (e.g., gluteus maximus and hamstrings) provide vertical support to the body and generate forward propulsion during the first half of the stance, which are the same roles of walking aids for patients with paraplegia during ambulation [30,31]. Based on these findings, we hypothesized that a reduction in upper extremity loading could be achieved during ambulation with an RGO by providing hip actuation with proper magnitude and timing. 


\section{Methods}

We investigated how upper extremity loading changed during ambulation when hip assistive forces were applied on the RGO compared with wearing an RGO with no actuation. To minimize the effect of adaptation and ensure safety, we recruited a participant who is competent at using RGO with walking aids. The study was approved by the Institutional Review Board at the Rehabilitation Engineering Research Institute, Korea Workers' Compensation and Welfare Services, and all study procedures were conducted in accordance with the approved study protocol.

\subsection{Participant}

One female individual with chronic (over 16 years after injury) T12 complete SCI classified as ASIA A participated in this study (age: 55 years, height: $1.64 \mathrm{~m}$, weight: $64.8 \mathrm{~kg}$ ). The subject has over 5 years of experience in using an RGO with crutches for ambulation. The participant provided written informed consent before participation and after the nature and possible consequences of the study were explained.

\subsection{Reciprocating Gait Orthosis}

The RGO used in this study was custom-made by a certified prosthetist and orthotist (CPO) for the participant, who has been using it for her rehabilitative training for over 5 years (Figure 1 ).

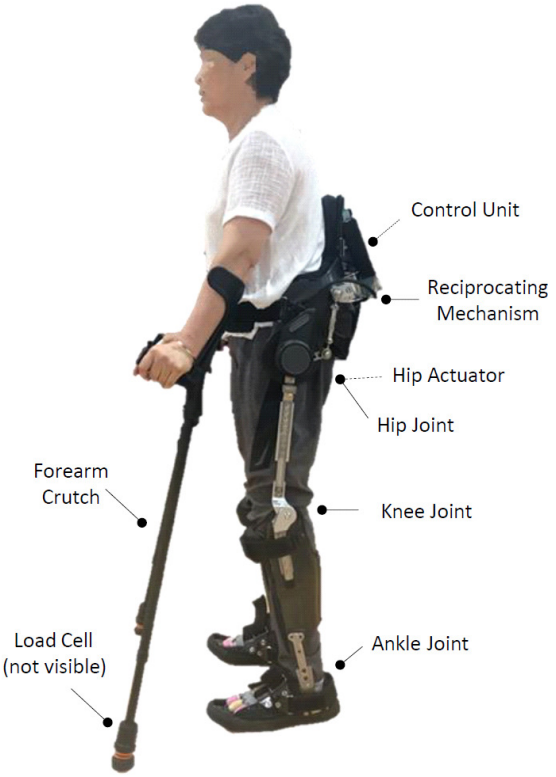

(a)
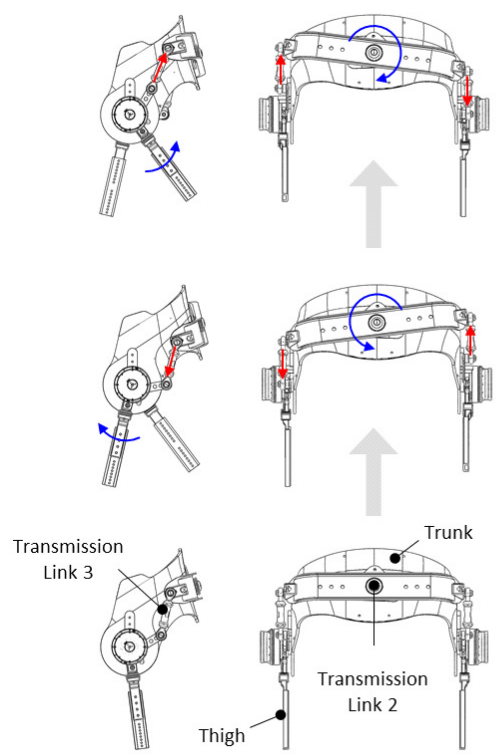

(b)
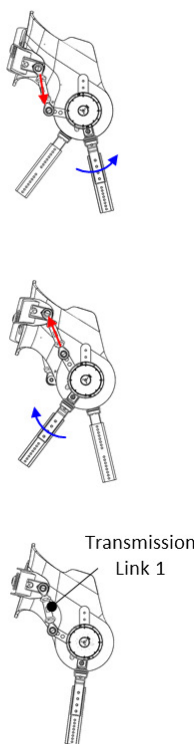

Figure 1. Reciprocating gait orthosis (a) and its reciprocating mechanism (b). Hip actuators were attached under the POWERED condition; they were not attached under the RGO condition.

The RGO had three degrees of freedom per leg to allow for physiological movements of the hip, knee, and ankle joints in the sagittal plane (i.e., hip flexion/extension, knee flexion/extension, and ankle plantarflexion/dorsiflexion). Both hip joints were mechanically coupled by a linkage system that allowed for reciprocal walking. The details of the reciprocating mechanism are illustrated in Figure 1b. For example, the extension of the right leg causes the rotation of transmission link 2 in a counter-clockwise direction by transmission link 1. The rotation of transmission link 2 results in the flexion of the left leg by transmission link 3 (see Video S1 for details). Solenoid-operated one-way clutches were used to lock the knee joints during the stance phase for collapse prevention. The joints were then unlocked during the swing phase to allow for knee flexion. Knee flexion during the swing phase reduces compensatory movements, such as hip elevation or circumduction 
induced by the lack of foot clearance for swinging the leg. The ankle joint had a limited range of motion to ensure the stability and safety of the patient during upright standing and ambulation. The participant and the RGO were closely fitted by harnesses at the trunk, shank, and feet. Forearm crutches were used as a walking aid during ambulation. The forearm crutches were instrumented with load cells to measure the crutch forces, defined as the applied reaction forces along the longitudinal axis of the crutches. The RGO with forearm crutches had a mass of $10.8 \mathrm{~kg}$, and the total mass including the hip actuators and control system was $14.0 \mathrm{~kg}$.

\subsection{Sensing and Control}

One inertial measurement unit (EBIMU-9DOF3, E2BOX) and two rotary potentiometers (WAL200, Contelec, Bern, Switzerland) were embedded in the RGO for measuring the trunk inclination with respect to gravity and the hip joint angles, respectively. A finite-state machine was designed to detect the five states of a gait cycle (Figure 2): standing (ST), double stance with left leg front (LST) (from left heel strike (LHS) to right toe off (RTO)), left stance extension (LEX) (from RTO to right leg heel strike (RHS)), double stance with right leg front (RST) (from RHS to left toe off (LTO)), and right stance extension (REX) (from LTO to LHS).

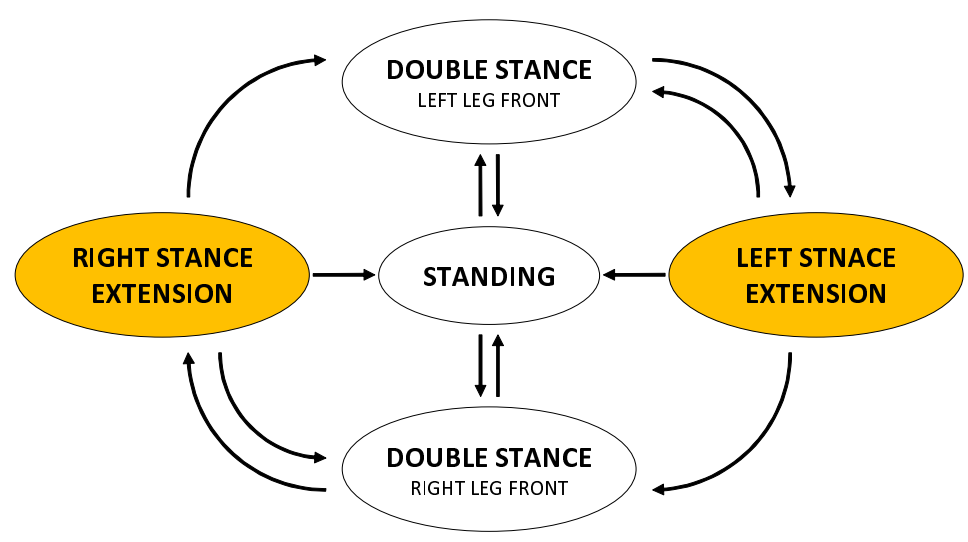

Figure 2. Finite-state machine for a reciprocating gait orthosis with hip actuation.

A transition was made from one state to another based on the values of hip angle and angular velocity. The values of hip angular velocity were obtained by taking the finitedifference derivative of the hip angles filtered by a first-order low-pass infinite impulse response filter. The state transition rules and actions are described in Table 1.

Table 1. State transition rules and actions.

\begin{tabular}{lll}
\hline State & Condition & Action \\
\hline ST & $\left(\left|\dot{\theta}_{l}\right|\right.$ and $\left.\left|\dot{\theta}_{r}\right| \leq \dot{\Theta}\right) \& \&\left(\left|\theta_{l}-\theta_{r}\right| \leq \Theta\right)$ & Friction compensation \\
LST & $\left(\left|\dot{\theta}_{l}\right|\right.$ and $\left.\left|\dot{\theta}_{r}\right| \leq \dot{\Theta}\right) \& \&\left(\left|\theta_{l}-\theta_{r}\right|>\Theta\right) \& \&\left(\left(\theta_{l}-\theta_{r}\right) \geq 0\right)$ & Friction compensation \\
LEX & $\left(\mathbf{S}==\right.$ LST $\& \&\left(\dot{\theta}_{l}<-\dot{\Theta}\right) \& \&\left(\dot{\theta}_{r}>\dot{\Theta}\right)$ & Friction compensation + Hip assistive force \\
RST & $\left(\left|\dot{\theta}_{l}\right|\right.$ and $\left.\left|\dot{\theta}_{r}\right| \leq \dot{\Theta}\right) \& \&\left(\left|\theta_{l}-\theta_{r}\right|>\Theta\right) \& \&\left(\left(\theta_{l}-\theta_{r}\right)<0\right)$ & Friction compensation \\
REX & $\left(\mathbf{S}==\right.$ RST $\& \&\left(\dot{\theta}_{l}>\dot{\Theta}\right) \& \&\left(\dot{\theta}_{r}<-\dot{\Theta}\right)$ & Friction compensation + Hip assistive force \\
\hline
\end{tabular}

S: current state, $\theta$ : hip angle, $\dot{\theta}$ : hip angular velocity, $\Theta$ : threshold of hip angle, $\dot{\Theta}$ : threshold of hip angular velocity. The subscripts $l$ and $r$ represents the left and right side, respectively. In this study, $\Theta$ and $\Theta$ were set to 10 .

\subsection{Hip Actuation}

A customized frameless, brushless DC motor (TBMS-6013-B, Kollmorgen, Radford, VA, USA) with harmonic drive transmission with a gear ratio of 50:1 (SHD-17-50-2SH, Harmonic Drive, Peabody, MA, USA) was used for hip actuation. In all states, the hip actuators were operated to eliminate the intrinsic friction behavior in the brushless DC motor with harmonic drive transmission to ensure the user's movement is not hindered. 
Assistive forces were added in the LEX and REX states to aid in stance extension and swing flexion. The assistive force profile was configured using multiple control parameters: onset timing $\left(\mathrm{T}_{o n}, \mathrm{~ms}\right)$, offset timing $\left(\mathrm{T}_{o f f}, \mathrm{~ms}\right)$, peak timing $\left(\mathrm{T}_{p k}, \%\right)$, peak width $\left(\mathrm{W}_{p k}, \%\right)$ and peak force $\left(\mathrm{M}_{p k}, \mathrm{Nm}\right)$ (Figure 3).

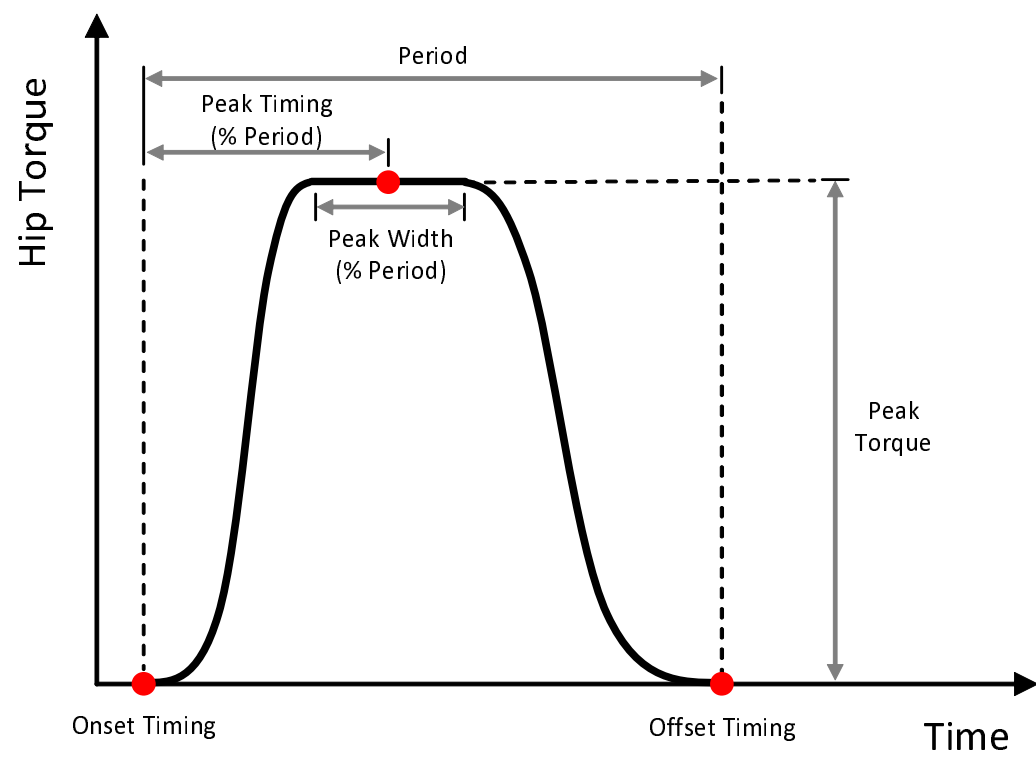

Figure 3. Hip assistive force profile.

These parameters were iteratively tuned until the participant could perform ambulation safely and comfortably. The final individualized parameters were as follows: $\mathrm{T}_{\text {on }}=0$, $\mathrm{T}_{o f f}=600, \mathrm{~T}_{p k}=30, \mathrm{~W}_{p k}=55$, and $\mathrm{M}_{p k}=-5$ for stance extension, $\mathrm{T}_{o n}=150, \mathrm{~T}_{\text {off }}=600$, $\mathrm{T}_{p k}=40, \mathrm{~W}_{p k}=8$, and $\mathrm{M}_{p k}=5$ for swing flexion. The resultant force profile indicates that assistive forces were generated for hip flexion during the stance phase and for hip extension during the swing phase (4th row in Figure 4). In addition, their sum, which affects trunk movement, was zero during the double stance state (from LHS to RTO and from RHS to LTO) to avoid impeding trunk flexion for the next step and negative value during the stance extension state (from RTO to RHS and from LTO to LHS) to assist trunk extension to initiate ambulation (bottom in Figure 4).

\subsection{Experimental Protocol}

The participant attended three separate sessions (familiarization, training, and testing) for the two device conditions (RGO and POWERED). The actuation and control system were attached to the RGO to provide powered hip assistance under the POWERED condition, but it was not attached under the RGO condition.

During the familiarization session for each device condition, static and dynamic alignments were performed by a CPO to ensure the RGO fit closely to the body of the participant. Under the POWERED condition, the magnitude and timing of hip actuation were individualized based not only on raw data analysis but also on oral feedback from the participant, the $\mathrm{CPO}$, and a physical therapist (PT). During the training session for each device condition, the participant performed independent ambulation with crutches under the supervision of the PT. The familiarization and training procedures for each device condition were conducted over a period of four weeks each (one hour a day, three times a week).

The testing session consisted of a single-day protocol performed three days after the end of the training session for each device condition to ensure recovery from fatigue. During these sessions, the participant underwent a $10 \mathrm{~m}$ walking test (10 MWT) with 10 trials at a self-selected walking speed. 

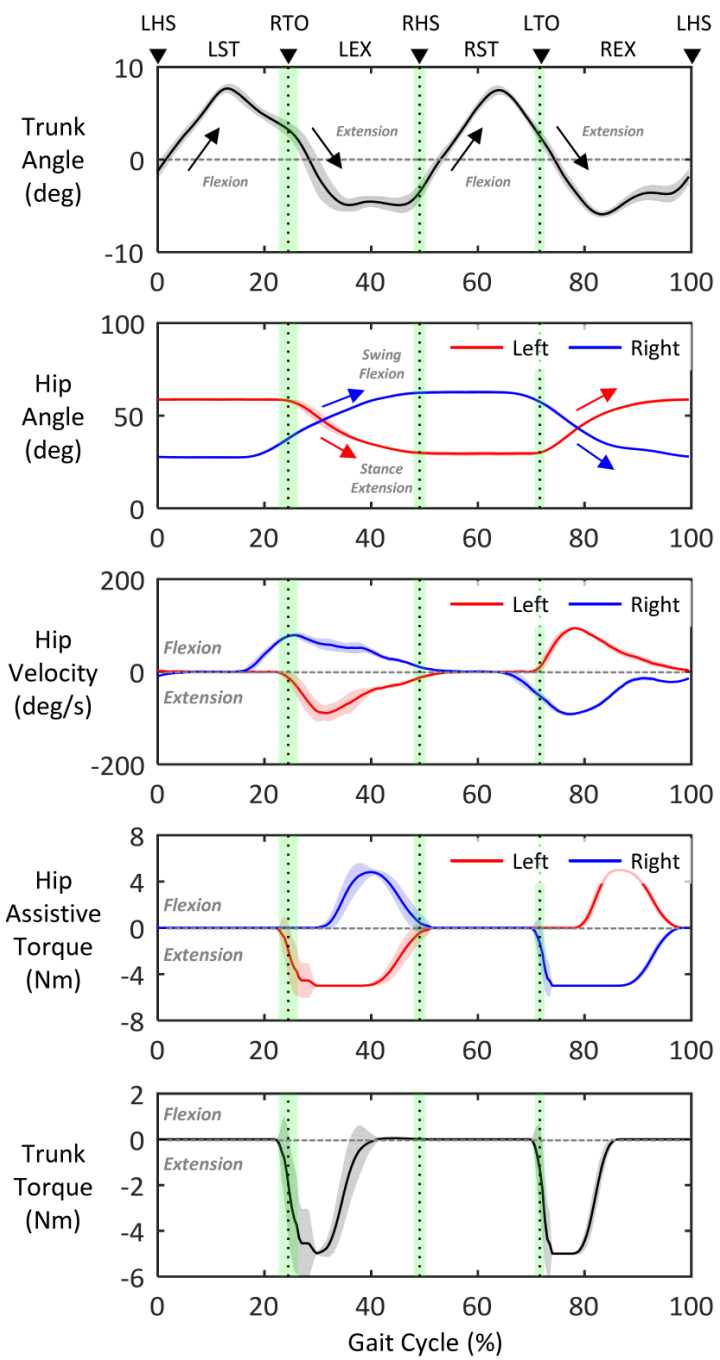

Figure 4. Measured data from the reciprocating gait orthosis during a gait cycle under the POWERED condition.

\subsection{Data Collection}

In all trials, the displacement of a sacral marker, ground reaction force (GRF) data, and crutch force data were collected simultaneously at a sampling frequency of $1.2 \mathrm{kHz}$ using a 3D motion capture system (Cortex 6.2.3, Motion Analysis, Santa Rosa, CA, USA) with twelve infrared cameras (Raptor 4s, Motion Analysis, Santa Rosa, CA, USA), two force plates (BP600900-2000, AMTI, Watertown, MA, USA), and two load cells (USL08-H6-2KN, Tec Gihan, Kyoto, Japan).

\subsection{Data Analysis}

\subsubsection{Center of Mass Trajectory}

The sacral marker method was used to estimate the displacement and velocity of the CoM during ambulation. This method involves measuring the displacement of a single reflective marker on the trunk or pelvis. It is based on the assumption that the movement of the trunk or pelvis mimics that of the CoM because of the close proximity to the actual location of the CoM [32-34].

Although the segmental analysis method is commonly used and regarded as the gold standard in CoM measurements, it is prone to inaccuracies because of the high variability of anthropometric characteristics among patients with paraplegia and between patients with SCI and average able-bodied subjects [35]. 


\subsubsection{Spatio-Temporal Gait Parameters}

GRF data were used to identify gait cycles, defined as the interval between two consecutive heel strikes of the same leg, and to segment gait phases. The magnitude of the GRF data was normalized by body weight. Stride length and stride time were determined based on the CoM displacement in the forward direction and by the duration time of the gait cycle, respectively. Gait speed was calculated by dividing stride length by stride time.

\subsubsection{Upper Extremity Loading}

Upper extremity loading during ambulation was assessed by measuring crutch forces. Peak upper extremity loading was defined as the maximum value among the force data measured from both crutches during a gait cycle. Average upper extremity loading was calculated by integrating the force data collected in both crutches during a gait cycle and dividing by the number of samples. To examine the effect of changes in the spatio-temporal gait parameters, we computed the average upper extremity loading per unit distance and per unit time by dividing the average upper extremity loading by stride length and stride time, respectively. The values of upper extremity loading were normalized to the participant's body weight.

\subsubsection{Statistics}

The mean and standard deviation of all collected and post-processed data were calculated for each device condition. According to the results of Shapiro-Wilk tests, all the data followed a normal distribution $(p>0.05)$. Therefore, we used paired Student $t$-tests to compare the mean values of both device conditions. Statistical analyses were performed using SPSS 18.0 (SPSS Inc., Chicago, IL, USA). A p-value of less than 0.05 was considered statistically significant.

\section{Results}

Measured data from the RGO during a gait cycle under the POWERED condition are illustrated in Figure 4. Figure 5 shows all the data measured by the 3D motion capture system during a single gait cycle under both device conditions.

\subsection{Gait Pattern}

The subject walked with a 4-point gait pattern, where the crutches and legs advanced reciprocally under all device conditions (Figure 6). More specifically, after each heel strike, the trunk leaned forward to move the body weight forward while the crutch on the leading leg side advanced (from LHS to RTO and from RHS to LTO in Figure 6). When the crutch came into contact with the ground, the subject performed trunk extension to generate stance extension of leading leg and swing flexion of trailing leg by using the reciprocating mechanism. At the same time, the subject pushed the crutch on the trailing leg side against the ground to transfer the body weight laterally from the trailing to the leading leg side (from RTO to RHS and from LTO to LHS in Figure 6) (see Video S1 for details). At this time, the hip assistive forces shown in Figure 4 were applied to the RGO under the POWERED condition.

\subsection{Spatio-Temporal Gait Parameters}

No statistically significant differences were found between both devices conditions in gait speed (RGO: $0.382 \pm 0.021$ and POWERED: $0.363 \pm 0.014 \mathrm{~ms}^{-1}$ ) and the stride length (RGO: $0.856 \pm 0.051$ and POWERED: $0.860 \pm 0.042 \mathrm{~m}$ ) (Figure 7). 

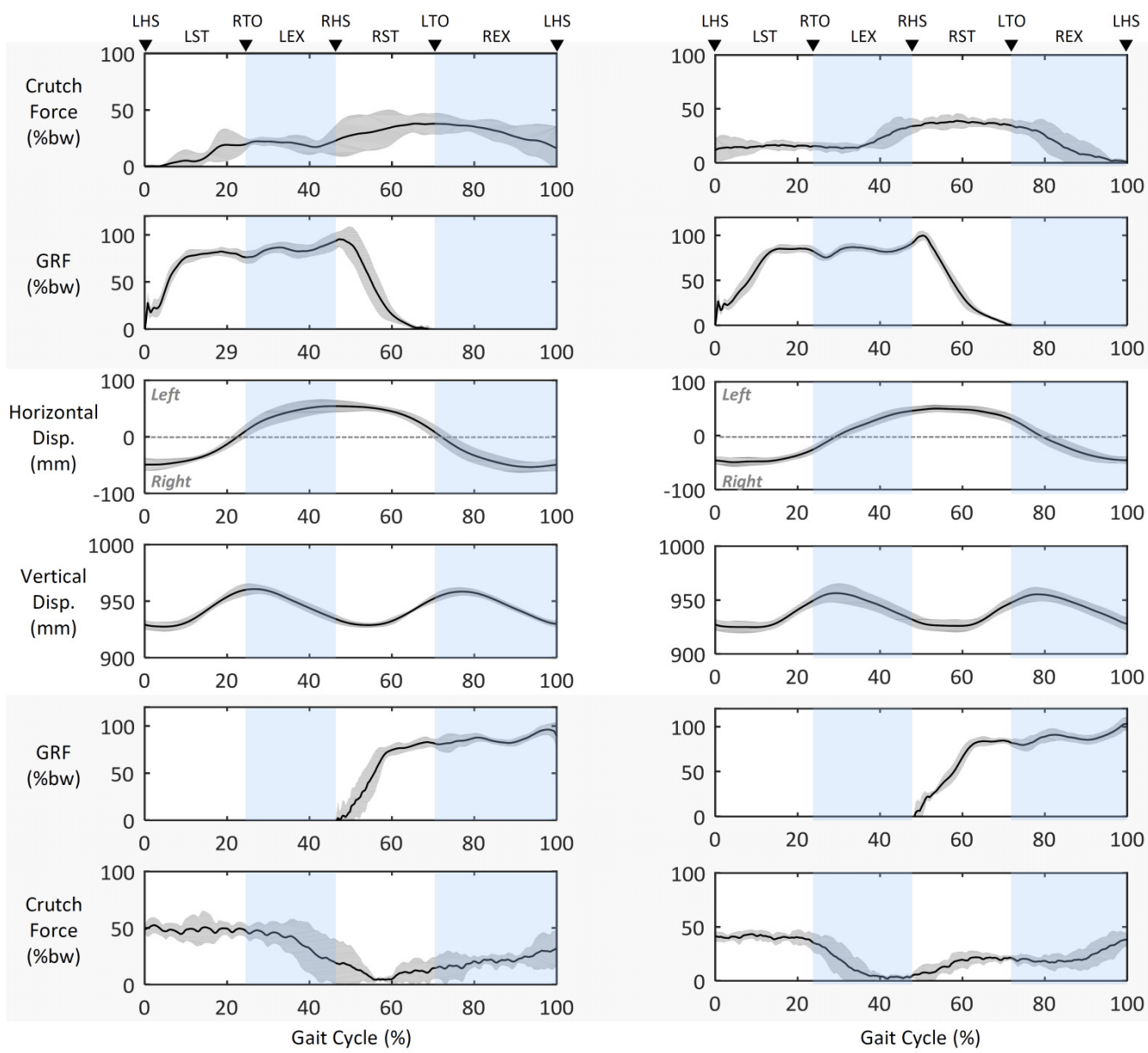

Figure 5. Center of mass trajectory, ground reaction force, and crutch force for each side during a gait cycle under the RGO condition (left) and the POWERED condition (right).
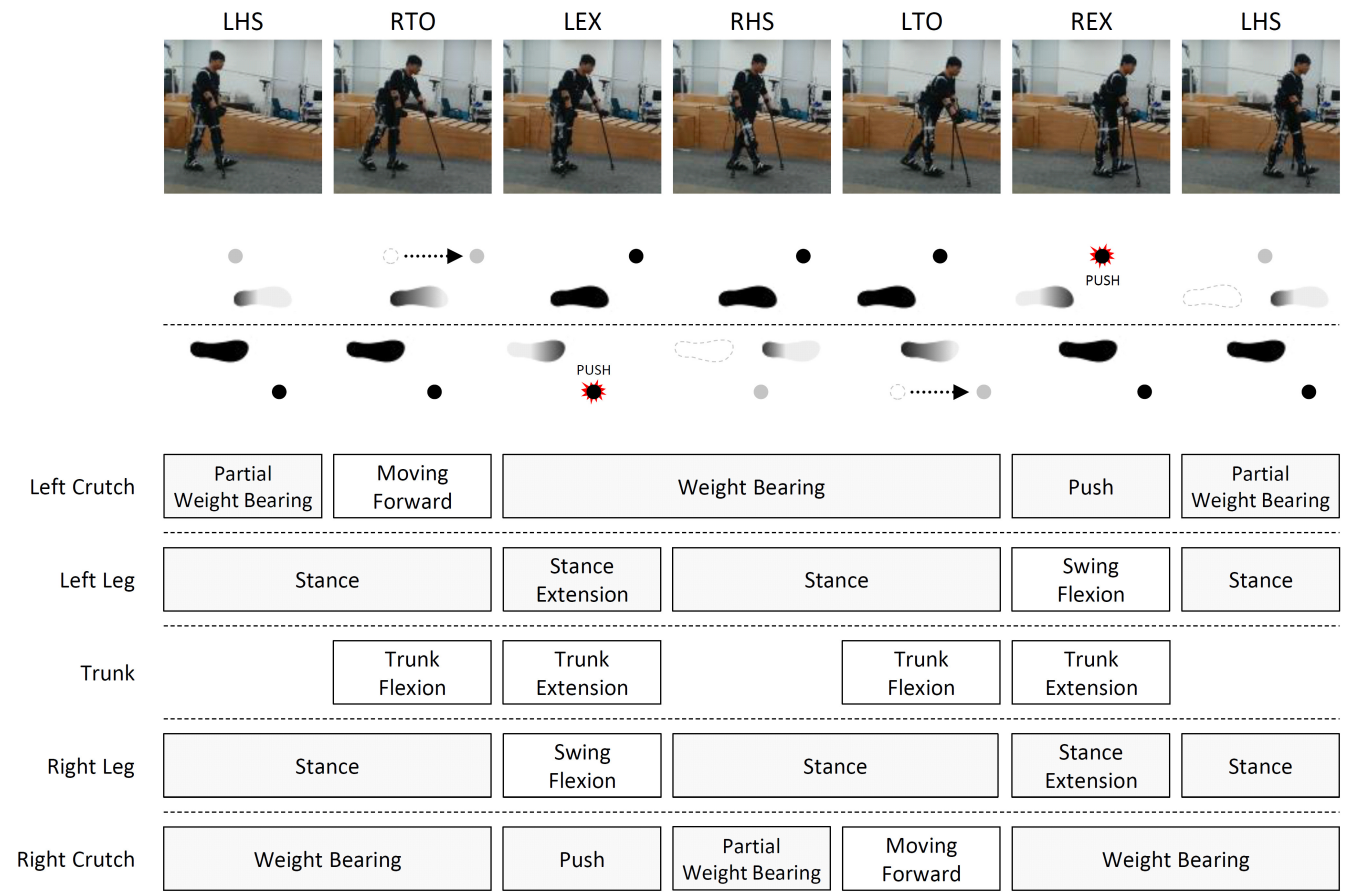

Figure 6. Sequence pattern of 4-point gait strategy. 


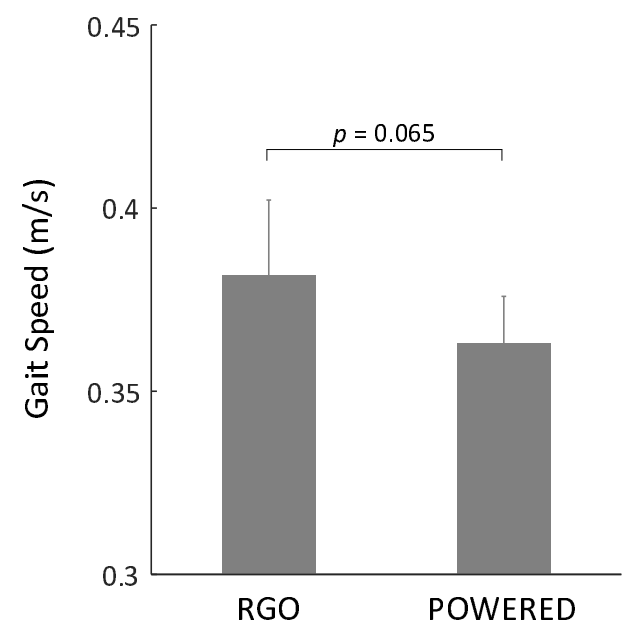

(a)

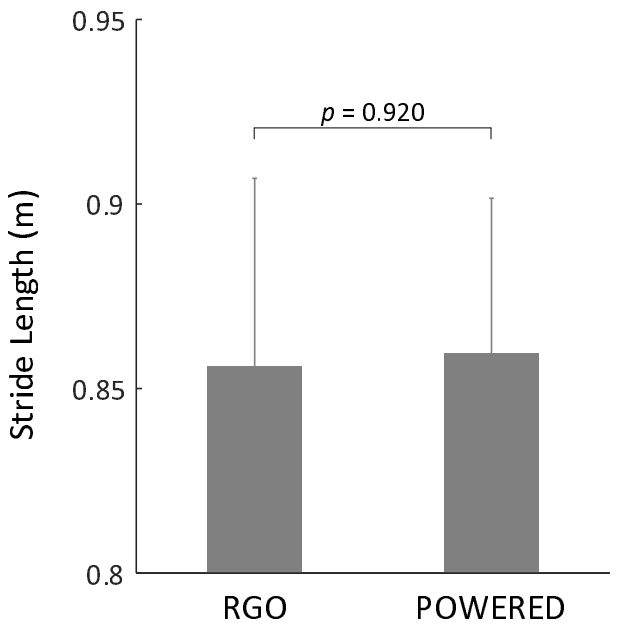

(b)

Figure 7. Spatiotemporal gait parameters: (a) gait speed and (b) stride length. Bars and error bars are means and standard deviations, respectively.

\subsection{Upper Extremity Loading}

Significant differences were observed in peak upper extremity loading and average upper extremity loading. Compared with the RGO condition, the peak and average upper extremity loadings under the POWERED condition were lower by 15.39\% (RGO: $0.535 \pm 0.084$ and POWERED: $0.453 \pm 0.054 \% \mathrm{bw}$ ) and by $14.62 \%$ (RGO: $0.262 \pm 0.043$ and POWERED: $0.224 \pm 0.029 \% \mathrm{bw}$ ), respectively (Figure 8). Considering the changes in spatio-temporal gait parameters, the average upper extremity loading per unit distance and per unit time were lower under the POWERED condition by $15.21 \%$ (RGO: $0.307 \pm 0.056$ and POWERED: $0.260 \pm 0.034 \% \mathrm{bw} \cdot \mathrm{m}^{-1}$ ) and by $21.19 \%$ (RGO: $0.120 \pm 0.020$ and POWERED: $0.094 \pm 0.011 \% \mathrm{bw} \cdot \mathrm{s}^{-1}$ ), respectively, under the RGO condition (Figure 9).

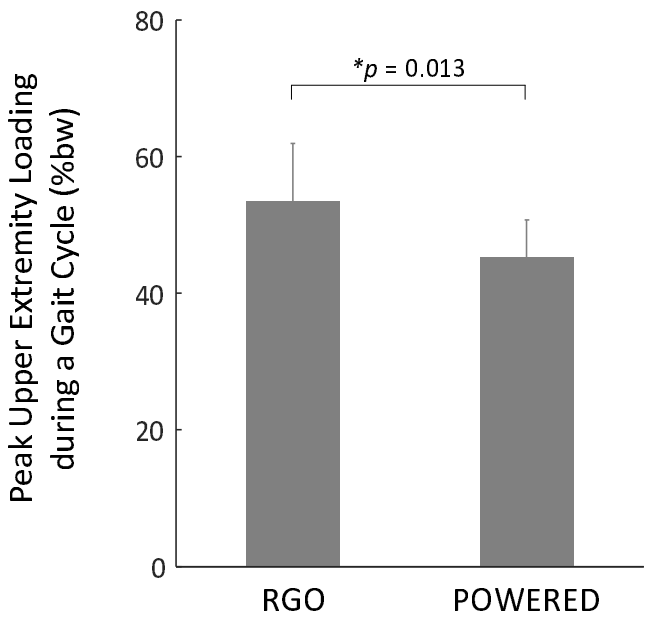

(a)

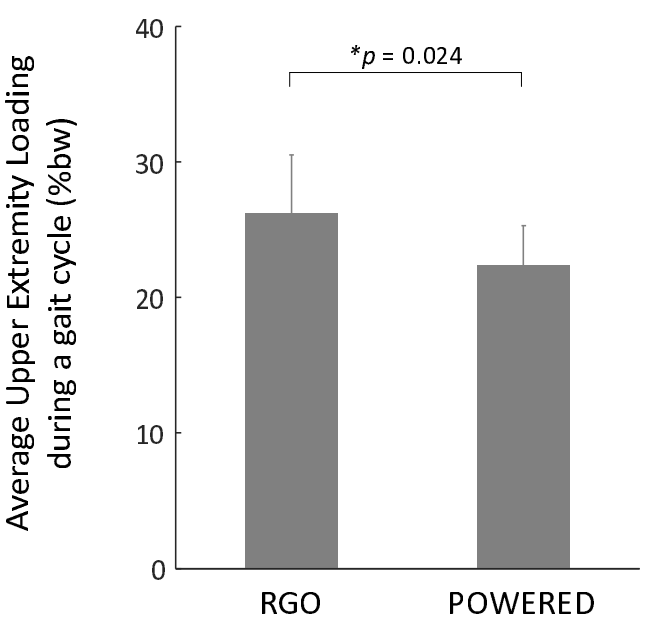

(b)

Figure 8. Peak (a) and average (b) upper extremity loading during a gait cycle. Bars are means, error bars are standard deviations, and asterisks denote statistical significance. 


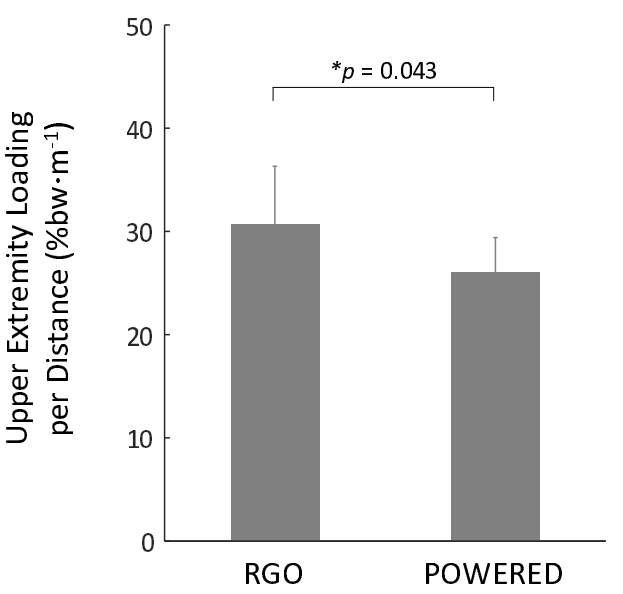

(a)

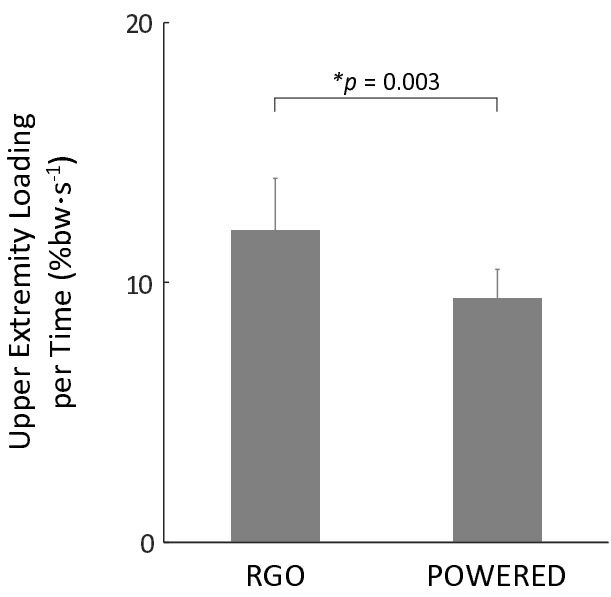

(b)

Figure 9. Average upper extremity loading per unit distance (a) and per unit time (b). Bars are means, error bars are standard deviations, and asterisks denote statistical significance.

\section{Discussion}

The results of this study support our hypothesis that the upper extremity loading of paraplegic patients during ambulation with an RGO can be reduced via individualized hip assistance. However, the reasons why hip actuation alleviates the upper extremity loading are still unclear. Based on our observations, this might be related to the movements of the $\mathrm{CoM}$. The trajectories of the CoM in the frontal plane for all device conditions are illustrated in Figure 10. Compared with the POWERED condition, the position of the apex of each step under the RGO condition was higher and biased toward the leading leg side. The fact that the apex of CoM trajectory is reached at the mid-stance of the gait cycle suggests that the stance flexion of the leading leg under the RGO condition was performed by the reciprocating mechanism when the body weight was on the leading leg side. This may have led to high crutch forces because the subject pushed the crutch until the body weight was shifted from the trailing leg to the leading leg side. In contrast, the stance flexion under the POWERED condition was performed via hip actuation when the body weight was approximately at the mid-point between both legs. This early stance flexion contributed to a lower apex height and shorter distance from the base to the apex, which may have resulted in the alleviation of upper extremity loading during ambulation.

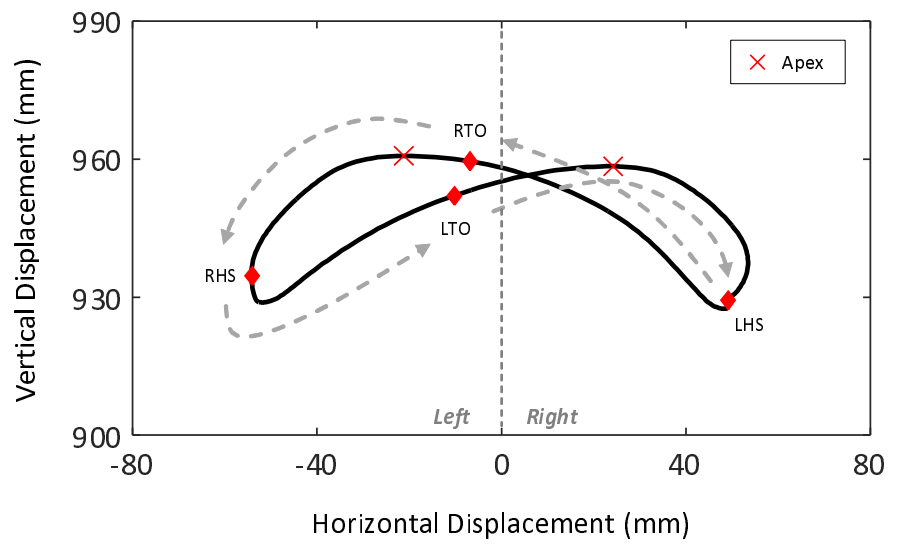

Figure 10. Cont. 


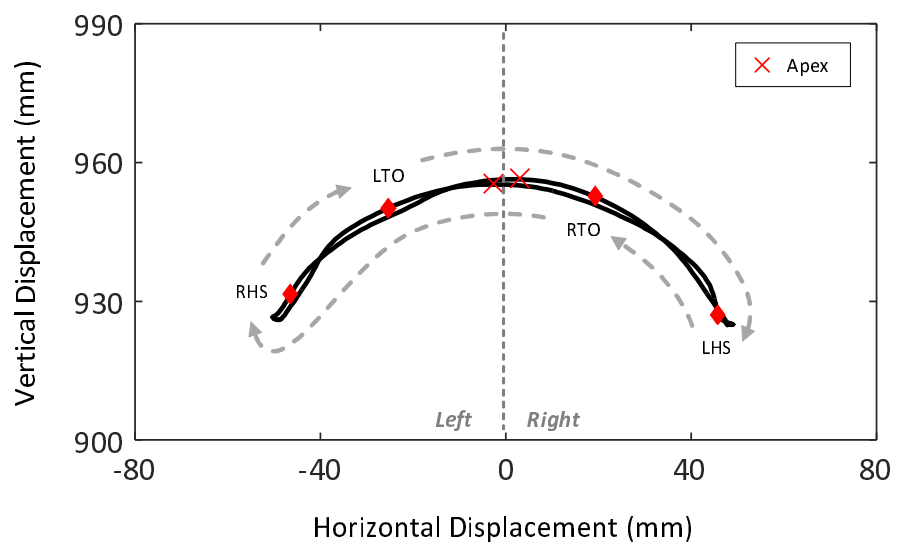

(b)

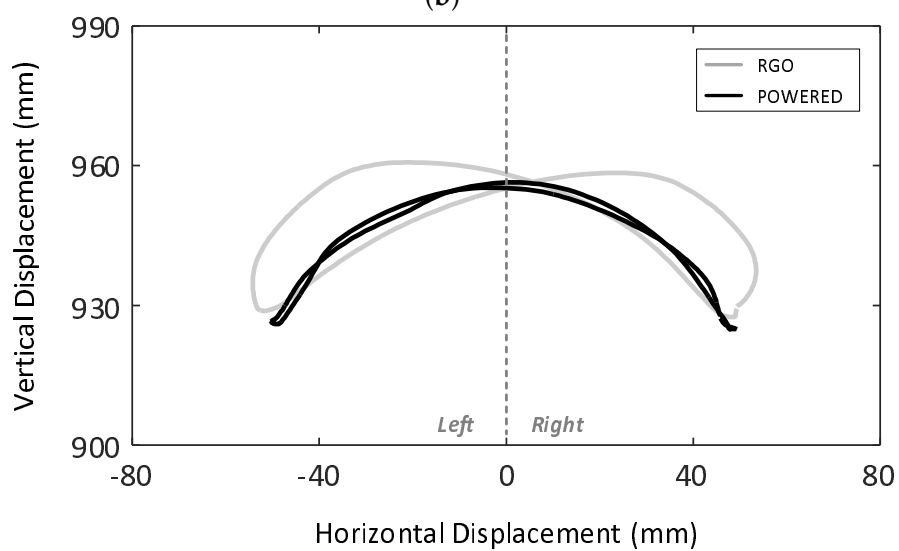

(c)

Figure 10. Center of mass trajectory in the frontal plane under the RGO condition (a) and the POWERED condition (b). Their trajectories are overlapped for comparison in (c).

\section{Conclusions}

In this study, we investigated the effect of hip actuation on the upper extremity loading induced by crutches when ambulating with an RGO. Our results show that the upper extremity loading of a patient with SCI may be reduced via individualized hip actuation. We believe that providing a substantial reduction in upper extremity loading during ambulation holds promise to promote long-term RGO usage and allow patients with paraplegia to perform frequent and intensive rehabilitation training. As a single case report, our findings may not be applicable to a larger population. Thus further research is required to assess the effects of individualized hip actuation according to the level of injury, level of mobility, level of proficiency with orthoses, and gait strategy.

Supplementary Materials: The following is available online at https:/ /youtu.be/EqNAXkTa0FA. Video S1: Reciprocating Gait Orthosis \& 4-Point Gait Strategy.

Author Contributions: Conceptualization, J.-W.L. and J.B.; methodology, J.-W.L. and J.B.; software, J.-W.L., Y.H. and J.B.; validation, J.-W.L. and J.B.; formal analysis, J.-W.L. and J.B.; investigation, J.-W.L. and H.C.; resources, J.-W.L., J.B., H.-J.C. and C.K.; data curation, J.L and J.B.; writing-original draft preparation, J.-W.L.; writing-review and editing, J.-W.L., J.B., H.-J.C., C.K., Y.H., H.C. and G.K.; visualization, J.-W.L.; supervision, H.C. and G.K.; project administration, J.-W.L. and H.C.; funding acquisition, H.C. All authors have read and agreed to the published version of the manuscript.

Funding: This work was supported by the Korea Medical Device Development Fund grant funded by the Korea government (the Ministry of Science and ICT, the Ministry of Trade, Industry and Energy, the Ministry of Health \& Welfare, Republic of Korea, the Ministry of Food and Drug Safety) (Project Number: 202013B01). 
Institutional Review Board Statement: The study was conducted according to the guidelines of the Declaration of Helsinki, and approved by the Institutional Review Board of the Rehabilitation Engineering Research Institute, Korea Workers' Compensation \& Welfare Service (RERI-IRB-200318-2).

Informed Consent Statement: Written informed consent has been obtained from the patient to publish this paper.

Data Availability Statement: The data presented in this study are available on request from the corresponding author on reasonable request.

Acknowledgments: The authors would like to thank Jungsun Kang, Bora Jeong and Yoonhee Chang for their assistance with experiments and our participant who gave her time for this study.

Conflicts of Interest: The authors declare no conflict of interest.

\section{References}

1. Ditunno, P.; Patrick, M.; Stineman, M.; Ditunno, J. Who wants to walk? Preferences for recovery after SCI: A longitudinal and cross-sectional study. Spinal Cord 2008, 46, 500-506. [CrossRef] [PubMed]

2. Sezer, N.; Akkus, S.; Uğurlu, F.G. Chronic complications of spinal cord injury. World J. Orthop. 2015, 6, 24. [CrossRef] [PubMed]

3. Karimi, M.T. What are the next steps in designing an orthosis for paraplegic subjects? Int. J. Prev. Med. 2012, 3, 145. [PubMed]

4. Douglas, R.; Larson, P.F.; McCall, R.E. The LSU reciprocation-gait orthosis. Orthopedics 1983, 6, 834-839. [CrossRef]

5. Jefferson, R.; Whittle, M. Performance of three walking orthoses for the paralysed: A case study using gait analysis. Prosthetics Orthot. Int. 1990, 14, 103-110. [CrossRef]

6. Whittle, M.; Cochrane, G.; Chase, A.; Copping, A.; Jefferson, R.; Staples, D.; Fenn, P.; Thomas, D. A comparative trial of two walking systems for paralysed people. Spinal Cord 1991, 29, 97-102. [CrossRef]

7. Franceschini, M.; Baratta, S.; Zampolini, M.; Loria, D.; Lotta, S. Reciprocating gait orthoses: A multicenter study of their use by spinal cord injured patients. Arch. Phys. Med. Rehabil. 1997, 78, 582-586. [CrossRef]

8. Tashman, S.; Zajac, F.E.; Perkash, I. Modeling and Simulation of Paraplegic Ambulation in a Reciprocating Gait Orthosis. J. Biomech. Eng. 1995, 117, 300-308. [CrossRef]

9. Baardman, G.; IJzerman, M.J.; Hermen, H.; Veltink, P.H.; Boom, H.; Zilvold, G. The influence of the reciprocal hip joint link in the Advanced Reciprocating Gait Orthosis on standing performance in paraplegia. Prosthetics Orthot. Int. 1997, 21, 210-221. [CrossRef]

10. Dall, P.; Müller, B.; Stallard, I.; Edwards, J.; Granat, M.H. The functional use of the reciprocal hip mechanism during gait for paraplegic patients walking in the Louisiana State University reciprocating gait orthosis. Prosthetics Orthot. Int. 1999, 23, 152-162. [CrossRef]

11. Johnson, W.B.; Fatone, S.; Gard, S.A. Walking mechanics of persons who use reciprocating gait orthoses. J. Rehabil. Res. Dev. 2009, $46,436-446$.

12. Merritt, J.; Miller, N.; Hanson, T. Preliminary Studies of Energy Expenditures in Paraplegics Using Swing-Thru and Reciprocating Gait Patterns. Arch. Phys. Med. Rehabil. 1983, 64, 510

13. Sykes, L.; Edwards, J.; Powell, E.S.; Ross, E.R.S. The reciprocating gait orthosis: Long-term usage patterns. Arch. Phys. Med. Rehabil. 1995, 76, 779-783. [CrossRef]

14. Scivoletto, G.; Petrelli, A.; Di Lucente, L.; Giannantoni, A.; Fuoco, U.; D’Ambrosio, F.; Filippini, V. One year follow up of spinal cord injury patients using a reciprocating gait orthosis: Preliminary report. Spinal Cord 2000, 38, 555-558. [CrossRef]

15. Requejo, P.S.; Wahl, D.P.; Bontrager, E.L.; Newsam, C.J.; Gronley, J.K.; Mulroy, S.J.; Perry, J. Upper extremity kinetics during Lofstrand crutch-assisted gait. Med. Eng. Phys. 2005, 27, 19-29. [CrossRef]

16. Rasouli, F.; Reed, K.B. Walking assistance using crutches: A state of the art review. J. Biomech. 2020, 98, 109489. [CrossRef] [PubMed]

17. Melis, E.; Torres-Moreno, R.; Barbeau, H.; Lemaire, E. Analysis of assisted-gait characteristics in persons with incomplete spinal cord injury. Spinal Cord 1999, 37, 430-439. [CrossRef]

18. Solomonow, M.; Baratta, R.; Hirokawa, S.; Rightor, N.; Walker, W.; Beaudette, P.; Shoji, H. The RGO generation II: Muscle stimulation powered orthosis as a practical walking system for thoracic paraplegics. Orthopedics 1989, 12, 1309-1315. [CrossRef] [PubMed]

19. Ferrarin, M.; Pedotti, A.; Boccardi, S.; Palmieri, R. Biomechanical assessment of paraplegic locomotion with hip guidance orthosis (HGO). Clin. Rehabil. 1993, 7, 303-308. [CrossRef]

20. Slavens, B.A.; Sturm, P.F.; Bajournaite, R.; Harris, G.F. Upper extremity dynamics during Lofstrand crutch-assisted gait in children with myelomeningocele. Gait Posture 2009, 30, 511-517. [CrossRef]

21. Lal, S. Premature degenerative shoulder changes in spinal cord injury patients. Spinal Cord 1998, 36, 186-189. [CrossRef] [PubMed]

22. Pennycott, A.; Wyss, D.; Vallery, H.; Klamroth-Marganska, V.; Riener, R. Towards more effective robotic gait training for stroke rehabilitation: A review. J. Neuroeng. Rehabil. 2012, 9, 1-13. [CrossRef] [PubMed] 
23. Molteni, F.; Gasperini, G.; Cannaviello, G.; Guanziroli, E. Exoskeleton and end-effector robots for upper and lower limbs rehabilitation: Narrative review. PMER 2018, 10, S174-S188. [CrossRef]

24. Miller, L.E.; Zimmermann, A.K.; Herbert, W.G. Clinical effectiveness and safety of powered exoskeleton-assisted walking in patients with spinal cord injury: Systematic review with meta-analysis. Med. Devices 2016, 9, 455. [CrossRef] [PubMed]

25. Zeilig, G.; Weingarden, H.; Zwecker, M.; Dudkiewicz, I.; Bloch, A.; Esquenazi, A. Safety and tolerance of the ReWalk ${ }^{\mathrm{TM}}$ exoskeleton suit for ambulation by people with complete spinal cord injury: A pilot study. J. Spinal Cord Med. 2012, 35, 96-101. [CrossRef]

26. Aach, M.; Cruciger, O.; Sczesny-Kaiser, M.; Höffken, O.; Meindl, R.C.; Tegenthoff, M.; Schwenkreis, P.; Sankai, Y.; Schildhauer, T.A. Voluntary driven exoskeleton as a new tool for rehabilitation in chronic spinal cord injury: A pilot study. Spine J. 2014, 14, 2847-2853. [CrossRef]

27. Farris, R.J.; Quintero, H.A.; Murray, S.A.; Ha, K.H.; Hartigan, C.; Goldfarb, M. A preliminary assessment of legged mobility provided by a lower limb exoskeleton for persons with paraplegia. IEEE Trans. Neural Syst. Rehabil. Eng. 2013, 22, 482-490. [CrossRef]

28. Kwon, S.H.; Lee, B.S.; Lee, H.J.; Kim, E.J.; Lee, J.A.; Yang, S.P.; Kim, T.Y.; Pak, H.R.; Kim, H.K.; Kim, H.Y.; et al. Energy efficiency and patient satisfaction of gait with knee-ankle-foot orthosis and robot (ReWalk)-assisted gait in patients with spinal cord injury. Ann. Rehabil. Med. 2020, 44, 131-141. [CrossRef]

29. Asselin, P.; Knezevic, S.; Kornfeld, S.; Cirnigliaro, C.; Agranova-Breyter, I.; Bauman, W.A. Heart rate and oxygen demand of powered exoskeleton-assisted walking in persons with paraplegia. J. Rehabil. Res. Dev. 2015, 52, 147. [CrossRef]

30. Neptune, R.; Zajac, F.; Kautz, S. Muscle force redistributes segmental power for body progression during walking. Gait Posture 2004, 19, 194-205. [CrossRef]

31. Liu, M.Q.; Anderson, F.C.; Pandy, M.G.; Delp, S.L. Muscles that support the body also modulate forward progression during walking. J. Biomech. 2006, 39, 2623-2630. [CrossRef] [PubMed]

32. Eng, J.J.; Winter, D. Estimations of the horizontal displacement of the total body centre of mass: Considerations during standing activities. Gait Posture 1993, 1, 141-144. [CrossRef]

33. Gard, S.A.; Miff, S.C.; Kuo, A.D. Comparison of kinematic and kinetic methods for computing the vertical motion of the body center of mass during walking. Hum. Mov. Sci. 2004, 22, 597-610. [CrossRef] [PubMed]

34. Yang, F.; Pai, Y.C. Can sacral marker approximate center of mass during gait and slip-fall recovery among community-dwelling older adults? J. Biomech. 2014, 47, 3807-3812. [CrossRef]

35. Thirunarayan, M.A.; Kerrigan, D.C.; Rabuffetti, M.; Della Croce, U.; Saini, M. Comparison of three methods for estimating vertical displacement of center of mass during level walking in patients. Gait Posture 1996, 4, 306-314. [CrossRef] 